Proceedings of the Edinburgh Mathematical Society (2002) 45, 349-352 (C)

DOI:10.1017/S0013091500000043 Printed in the United Kingdom

\title{
2-LOCAL ISOMETRIES OF SOME OPERATOR ALGEBRAS
}

\author{
LAJOS MOLNÁR \\ Institute of Mathematics and Informatics, University of Debrecen, \\ 4010 Debrecen, PO Box 12, Hungary (molnarl@math.klte.hu)
}

(Received 11 January 2000)

\begin{abstract}
As a consequence of the main result of the paper we obtain that every 2-local isometry of the $C^{*}$-algebra $B(H)$ of all bounded linear operators on a separable infinite-dimensional Hilbert space $H$ is an isometry. We have a similar statement concerning the isometries of any extension of the algebra of all compact operators by a separable commutative $C^{*}$-algebra. Therefore, on those $C^{*}$-algebras the isometries are completely determined by their local actions on the two-point subsets of the underlying algebras.
\end{abstract}

Keywords: operator algebra; isometry; local isometry

AMS 2000 Mathematics subject classification: Primary 47B49

In a series of papers (see $[\mathbf{1}, \mathbf{6}-\mathbf{8}]$ and the references cited therein) we investigated the automorphism groups and the isometry groups of operator algebras from the point of view of how they are determined by their local actions. Our investigations were motivated by the paper by Kadison [3] on local derivations and by a problem of Larson in [5] initiating the study of local automorphisms of Banach algebras. In those papers we considered the following question. When is it true that any local automorphism, that is, any linear transformation which pointwise equals an automorphism (this automorphism may, of course, differ from point to point), is an automorphism? If the answer to this question is affirmative, then we say that the automorphism group of the algebra in question is algebraically reflexive.

It is easy to see that if we drop the assumption of linearity of our local maps, then the corresponding statements are no longer true. However, if, instead of linearity plus locality, we assume the so-called 2-locality, then we can obtain positive results. Motivated by the paper by Kowalski and Slodkowski [4], the concept of 2-locality was introduced by Šemrl, who obtained the first results on 2-local automorphisms and 2-local derivations [9]. Besides the automorphism group and the derivation algebra, probably the third most important class of transformations on an operator algebra is the isometry group which reflects the geometrical properties of the underlying algebra. This motivates us to consider the local properties of this group. The main result of this paper is that in certain operator algebras, the 2-local isometries are in fact automatically linear. As a consequence, it follows that on some important $C^{*}$-algebras, every 2 -local isometry is an isometry. 
Let $\mathcal{X}$ be a Banach space. By an isometry of $\mathcal{X}$ here we mean a surjective linear normpreserving map of $\mathcal{X}$. A mapping $\phi: \mathcal{X} \rightarrow \mathcal{X}$ (no linearity is assumed) is called a 2-local isometry of $\mathcal{X}$ if for every $A, B \in \mathcal{X}$ there is an isometry $\phi_{A, B}$ of $\mathcal{X}$, depending on $A$ and $B$, such that $\phi_{A, B}(A)=\phi(A)$ and $\phi_{A, B}(B)=\phi(B)$.

In what follows $H$ is a complex infinite-dimensional separable Hilbert space. Denote by $B(H), C(H)$ and $F(H)$ the algebra of all bounded linear operators on $H$, the algebra of all compact operators on $H$ and the algebra of all bounded finite rank operators on $H$, respectively.

Our main result reads as follows.

Theorem 1. Let $\mathcal{A} \subset B(H)$ be a $C^{*}$-subalgebra which contains $C(H)$ and the identity I. If $\phi: \mathcal{A} \rightarrow \mathcal{A}$ is a 2-local isometry, then $\phi$ is linear.

Proof. From [6, Lemma 2.1] we know that any isometry $\phi$ of $\mathcal{A}$ is either of the form

$$
\phi(A)=U A V \quad(A \in \mathcal{A})
$$

or of the form

$$
\phi(A)=U A^{\mathrm{T}} V \quad(A \in \mathcal{A}),
$$

where $U, V \in B(H)$ are unitaries and the superscript ' $\mathrm{T}$ ' denotes the transpose with respect to an arbitrary but fixed complete orthonormal sequence in $H$. Now, let $\phi: \mathcal{A} \rightarrow$ $\mathcal{A}$ be a 2-local isometry of $\mathcal{A}$. By the form of the isometries of $\mathcal{A}$ it is easy to check that we have

$$
\operatorname{tr} \phi(A) \phi(B)^{*}=\operatorname{tr} A B^{*}
$$

for all finite-rank operators $A, B \in \mathcal{A}$ (here tr denotes the usual trace functional). By the linearity of tr this implies that

$$
\operatorname{tr}\left(\phi\left(A+A^{\prime}\right)-\phi(A)-\phi\left(A^{\prime}\right)\right) \phi(B)^{*}=\operatorname{tr}\left(\left(A+A^{\prime}\right)-A-A^{\prime}\right) B^{*}=0,
$$

and then we obtain

$$
\operatorname{tr}\left(\phi\left(A+A^{\prime}\right)-\phi(A)-\phi\left(A^{\prime}\right)\right)\left(\phi\left(A+A^{\prime}\right)-\phi(A)-\phi\left(A^{\prime}\right)\right)^{*}=0 .
$$

Consequently, it follows that $\phi\left(A+A^{\prime}\right)=\phi(A)+\phi\left(A^{\prime}\right)\left(A, A^{\prime} \in F(H)\right)$, that is, $\phi$ is additive on $F(H)$. Similarly, we find that $\phi$ is homogeneous. By the 2-local property of $\phi$, it follows that $\|\phi(A)-\phi(B)\|=\|A-B\|$ for every $A, B \in \mathcal{A}$. Therefore, $\phi$ is continuous on $\mathcal{A}$ and, since $F(H)$ is dense in $C(H)$, we infer that $\phi$ is linear on $C(H)$. So, the restriction of $\phi$ onto $C(H)$ is a linear 2-local isometry of $C(H)$. Clearly, $\phi$ is a linear map on $C(H)$ which preserves the rank of the finite-rank operators. The form of such transformations was studied in [2]. The statement [2, Theorem 1.1] characterizes the weakly continuous rank preservers on $B(H)$. An inspection of the proof presented there shows that the norm-continuous linear rank preservers on $C(H)$ are of the same form. Namely, we have operators $U, V \in B(H)$ such that $\phi$ is either of the form

$$
\phi(A)=U A V \quad(A \in C(H))
$$


or of the form

$$
\phi(A)=U A^{\mathrm{T}} V \quad(A \in C(H)) .
$$

Without loss of generality we can assume that $\phi$ is of the first form. Since $\phi$ preserves the norm of compact operators, it follows that $U$ and $V$ can be chosen to be an isometry and a coisometry, respectively. Moreover, if we pick an injective compact operator with dense range, referring to the local form of $\phi$ we obtain that $U, V$ are in fact unitaries. Let $T \in \mathcal{A}$ and let $P$ be an arbitrary rank-one projection. There exists an isometry $\phi_{P, T}$ of $\mathcal{A}$ such that

$$
\phi(P)=\phi_{P, T}(P) \quad \text { and } \quad \phi(T)=\phi_{P, T}(T) .
$$

Since $P$ is of rank one, it follows that $P T^{*} P=\lambda P$ for some $\lambda \in \mathbb{C}$. Now, we can compute

$$
\begin{aligned}
U P V \phi(T)^{*} U P V & =\phi(P) \phi(T)^{*} \phi(P)=\phi_{P, T}(P) \phi_{P, T}(T)^{*} \phi_{P, T}(P) \\
& =\phi_{P, T}\left(P T^{*} P\right)=\phi_{P, T}(\lambda P)=\lambda \phi_{P, T}(P)=\lambda U P V=U\left(P T^{*} P\right) V .
\end{aligned}
$$

Since $P$ was arbitrary, it follows that

$$
\phi(T)=U T V \quad(T \in \mathcal{A}) .
$$

This completes the proof of the statement.

From our theorem we immediately have the following corollary.

Corollary 2. Let $\mathcal{A}$ be as above. If the isometry group of $\mathcal{A}$ is algebraically reflexive, then every 2-local isometry of $\mathcal{A}$ is an isometry.

By the results in $[\mathbf{1}, \mathbf{6}]$ we can formulate the next two statements.

Corollary 3. Every 2-local isometry of $B(H)$ is an isometry.

The $C^{*}$-algebras appearing in the following corollary are the main objects of the celebrated Brown-Douglas-Fillmore (BDF) theory.

Corollary 4. Let $\mathcal{A}$ be the extension of $C(H)$ by a separable commutative $C^{*}$-algebra. Then every 2-local isometry of $\mathcal{A}$ is an isometry of $\mathcal{A}$.

The argument applied in the proof of our theorem and Sourour's result [10, Theorem 2] on the form of the isometries of norm ideals of $B(H)$ gives us our final result, which follows.

Corollary 5. If $\mathcal{I}$ is a minimal norm ideal in $B(H)$ different from the Hilbert-Schmidt class, then every 2-local isometry of $\mathcal{I}$ is an isometry.

Remark 6. Since every $*$-automorphism of the algebras discussed in the paper are isometries, one can easily obtain similar results concerning 2-local $*$-automorphisms.

Finally, we feel that, just as in the case of linear (1-)local isometries (see [8]), it would be interesting to investigate the 2-local isometries of other or more-general Banach spaces.

Acknowledgements. This research received support from the Hungarian National Foundation for Scientific Research (OTKA) under grant no. T-030082 F-019322. The author also received a grant from the Ministry of Education, Hungary, reg. no. FKFP 0304/1997. 


\section{References}

1. C. J. K. BAtty And L. MolnáR, On topological reflexivity of the groups of *automorphisms and surjective isometries of $B(H)$, Arch. Math. 67 (1996), 415-421.

2. J. C. Hou, Rank-preserving linear maps on B(X), Sci. China A 32 (1989), 929-940.

3. R. V. Kadison, Local derivations, J. Algebra 130 (1990), 494-509.

4. S. KowAlski AND Z. SlodKOWski, A characterization of multiplicative linear functionals in Banach algebras, Studia Math. 67 (1980), 215-223.

5. D. R. LARSOn, Reflexivity, algebraic reflexivity and linear interpolation, Am. J. Math. 110 (1988), 283-299.

6. L. MolnÁr, Reflexivity of the automorphism and isometry groups of $C^{*}$-algebras in BDF theory, Arch. Math. 74 (2000), 120-128.

7. L. MOLnÁR AND M. GYŐRY, Reflexivity of the automorphism and isometry groups of the suspension of $B(H)$, J. Funct. Analysis 159 (1998), 568-586.

8. L. Molnár AND B. Zalar, Reflexivity of the group of surjective isometries on some Banach spaces, Proc. Edinb. Math. Soc. 42 (1999), 17-36.

9. P. ŠEmRl, Local automorphisms and derivations on $B(H)$, Proc. Am. Math. Soc. 125 (1997), 2677-2680.

10. A. R. Sourour, Isometries of norm ideals of compact operators, J. Funct. Analysis 43 (1981), 69-77. 